\title{
Association Between The SNP Rs2154545 Polymorphism on The DyrkIA Gene and Intellectual Disability in Down Syndrome
}

\author{
Eva Chundrayetti ${ }^{1}$, Indra Ihsan ${ }^{2}$, Amel Yanis ${ }^{3}$ \\ \{echundrayetti@yahoo.com ${ }^{1}$ \} \\ Division of Children Health Sciences, Dr.M.Djamil Hospital/Faculty of Medicine, Universitas \\ Andalas ${ }^{1,2}$ \\ Division of Mental Health Sciences Dr.M.Djamil Hospital/Faculty of Medicine, Universitas Andalas ${ }^{3}$
}

\begin{abstract}
Down syndrome, or trisomy 21, is a genetic abnormality caused when chromosome 21 fails to separate during meiotic division, resulting in offspring with 47 chromosomes. People with this syndrome are known to have mild to severe intellectual disability. One of the pivotal genes on chromosome 21 is Dual-specificity tyrosinephosphorylation regulated kinase 1A (DyrkIA), and some of its polymorphisms (for example, SNP rs2154545) may cause phenotypic changes ina person with Down syndrome. The purpose of this study is to demonstrate whether SNP rs2154545 polymorphisms are associated with theDyrkIA gene and to analyze its relationship with intellectual disability in Down syndrome. This was a cross-sectional observational study that used blood samples from 39 subjects. All subjects were identified through Special School Kodya Padang, they were clinically proven to have Down syndrome, and the trisomy 21 diagnosis was confirmed by karyotyping analysis. Their DNA samples were analyzed using restriction fragment length polymorphism (RFLP), the samples were sequenced, and the contigs were qualitatively analyzed. Multiple alignment and BLASTNCBI was used to confirm the restriction result. The rs2154545 polymorphism on the DyrkIA gene was observed in the form of homozygote mutant alleles (AA) in $5.1 \%$ of all samples and heterozygote mutant alleles (GA) in $51.3 \%$ of all samples. The remaining samples were wild-type (43.6\%). Statistical analysis was performed, but there were no significant relationships $(\mathrm{p}>0.05)$ between gene polymorphism and intellectual disability. The rs 2154545 polymorphism on the DyrkIA gene was proven to exist in some people with Down syndrome, but there were no significant relationships between the polymorphism and every category of intellectual disability(that is, mild, borderline, moderate, and severe).
\end{abstract}

Keywords: Down Syndrome, DyrkIA Gene, Intellectual Disability, RS2154545 Polymorphism.

\section{Introduction}

Down syndrome (DS) is a major pediatric issue due to its impact on growth and development. Cognitive development is the most inhibited factor and causes hindrance in everyday life. People with DS need help and counseling from family, the community, and the country. DS is a genetic abnormality that result intellectual disability, hypotonia, facial dysmorphic, earlier onset of Alzheimer's disease, and other behavior disruptions. The incidence 
of DS in the United States is estimated to occur once in every 800-1000 birth. There are not yet any definitive incidence estimates in Indonesia; however, research by Indonesia University predicted that at least 300,000 new DS cases occur every year. Based on data from Dr. M. Djamil Hospital Padang, 95 DS cases occurred between 2009 and 2012, and 112 DS cases from 2013 to 2016 (Dr.M.Djamil Hospital Padang,2016).[1]

DS, also known as trisomy 21 , is caused by meiotic non-disjunction $(95.4 \%$ of all observed cases), Robertsonian translocation $(2.7 \%)$, mosaic non-disjunction $(0.7 \%)$, and rearrangement of the genetic material on chromosome 21 with other acrocentric chromosomes, such as chromosome 14 (2.0\%). Chromosome 21 has 200-400 genes. Etiology and pathology studies of DS have been focused on the extra copy of the region in the proximal position of chromosome 21 , mainly at 21q22.3. The distal region of the long arm of chromosome 21, about 10megabase pairs (Mb) in length, is known as the DS critical region (DSCR). Some of the genes in the DSCR are known to play a role in DS pathogenesis, such as Dual-specificity tyrosine phosphorylationregulated kinase (DyrkIA), which codes for superoxide dismutase 1 (SOD1) enzyme; cystathionine beta-synthase (CBS); and glycinamide ribonucleotide synthetase-aminoimidazole ribonucleotide synthetase-glycinamide formyltransferase (GARS-AIRS-GART). Gene duplication in the DSCR may give several depictions in main phenotypic abnormalities of DS. This evidence gives a significant contribution to DS pathogenesis, including phenotype abnormalities, such as morphology figure, hypotonia, and intellectual disability. [2][3]

Dyrk is a homolog from the Drosophila cerebellum that is responsible for neurogenesis. Dyrk has two specificities to tyrosine kinase and serine/threonine kinase, and it is regulated by phosphorylated tyrosine [4]. The latest research on the DyrkIA gene has shown that is located at $21 \mathrm{q} 22.3$, which is on the long arm of chromosome 21 . This location is $5.4 \mathrm{Mb}$ in size and contains 30 genes, based on the phenotype and genotype correlation in a partial trisomy 21 case study. A similar correlation study investigating partial monosomy 21 revealed the location of the DSCR. The DSCR is 1,2 Mb in size and contains 10 genes, including DyrkIA [5].

DyrkIAis overexpressed in DS. This overexpression plays an important role in brain degeneration and early-onset neurodegeneration, nerve fiber loss, and dementia in people with DS. Based on the identification of hundreds of genes that reformed by the DyrkIA overexpression, expression of cytosolic, cytoskeletal, and nuclear protein, including transcription factor and phosphorylation, it can be inferred that DyrkIA overexpression is the center of the multiple pathway deregulations in brain development and aging with structural and functional damage that leads to intellectual disability and dementia. DrykIA overexpression in DS contributes directly to early-onset neurofibril degeneration by hyperphosphorylation of the tau protein and indirectly by the phosphorylation of alternative splicing factor (ASF), which imbalances the 3R-tau and 4R-tau proteins (Wegiel, 2011).

Another study showed that MBN or DyrkIA have a role in the development of the central nervous system, neuron proliferation, neurogenesis, neuron differentiation, cell death, and synapse plasticity [4]. Its overexpression in the fetus brain of people who have DS supports the hypothesis of a role for MNB or DyrkIA in neuron development, which is the basis of the cognitive deficiency that is present in people with DS (Tejedor, 2011). Some studies have shown that DS is related to weaker short-term verbal memory capabilities and explicit long-term memory. However, explicit memory is slightly affected [5]. An infant with mild to moderate intellectual disability is usually able to master early raw motoric milestones at the right time, but an infant with DS usually shows latency in the early raw motoric mastery as the result of hypotonia [6]. Intellectual disability is rarely observed in an infant younger than 3 years old as the assessment instrument for this age range is not well correlated with later IQ tests. Formal IQ 
tests in school-age children are considered to be a better reflection of the child long-term cognitive abilities [6].

Recent studies observed that DyrkIAplays a role in the interference of early cognitive development and brain degeneration, which causes the intelligence disruption as reflected by IQ scores. The present study has been performed to elucidate the DyrkIA gene polymorphism SNP rs2154545 and its relationship to intelligence in DS.The objectives of this study are to demonstrate whether the SNP rs2154545 polymorphism occurs in theDyrkIA gene and to analyze its relationship with intellectual disability in DS.

\section{Materials and Methods}

This study was conducted using blood samples from 39 students from the Special School Kodya Padang who meet the inclusion criteria. Up to $4 \mathrm{cc}$ of blood was taken from each student's vein for cytogenic analysis and DNA extraction. Polymerase chain reaction (PCR) and sequencing were performed in a biomedical laboratory in the medical faculty atAndalas University, Padang and Macrogen, South Korea. A professional psychologist performed IQ testing.

\subsection{DNA Extraction (Genomic DNA Purification)}

Genomic DNA was isolated from $300 \mu$ l blood sample using the Genomic DNA Mini Kit (Blood/Cultured Cell) GB100(Geneaid). DNA isolation was performed by following the instructions in the kit manual, including sample preparation, cell lysis, DNA binding, washing and rinsing, and DNA elution.

\subsection{Rs2154545 (G>A) Genotyping}

Genotyping for the SNP Rs1254545 polymorphismon theDyrkIA gene (NCBI accession number: NG_009366.1) was performed using the restriction fragment length polymorphism (RFLP)-PCR method. PCR reagents consisted of: (1) $0.2 \mu \mathrm{M}$ deoxynucleoside triphosphates (dNTPs); (2) 100 ng genomic DNA; (3) 1.25 U HotStarTaq DNA Polymerase with its buffer;

(4) $0.5 \mu \mathrm{M}$ forward primer rs2154545-F (5'-GGATTCTGTAGTGGATATGTAGTGG-3'); and (5) $0.5 \mu \mathrm{M}$ reverse primer $\mathrm{rs} 2154545-\mathrm{R}$

(5'ACCCTGTTCCACGCTCAAAA-3'). All reagents werecombined to createa total volume of $25 \mu 1$. 


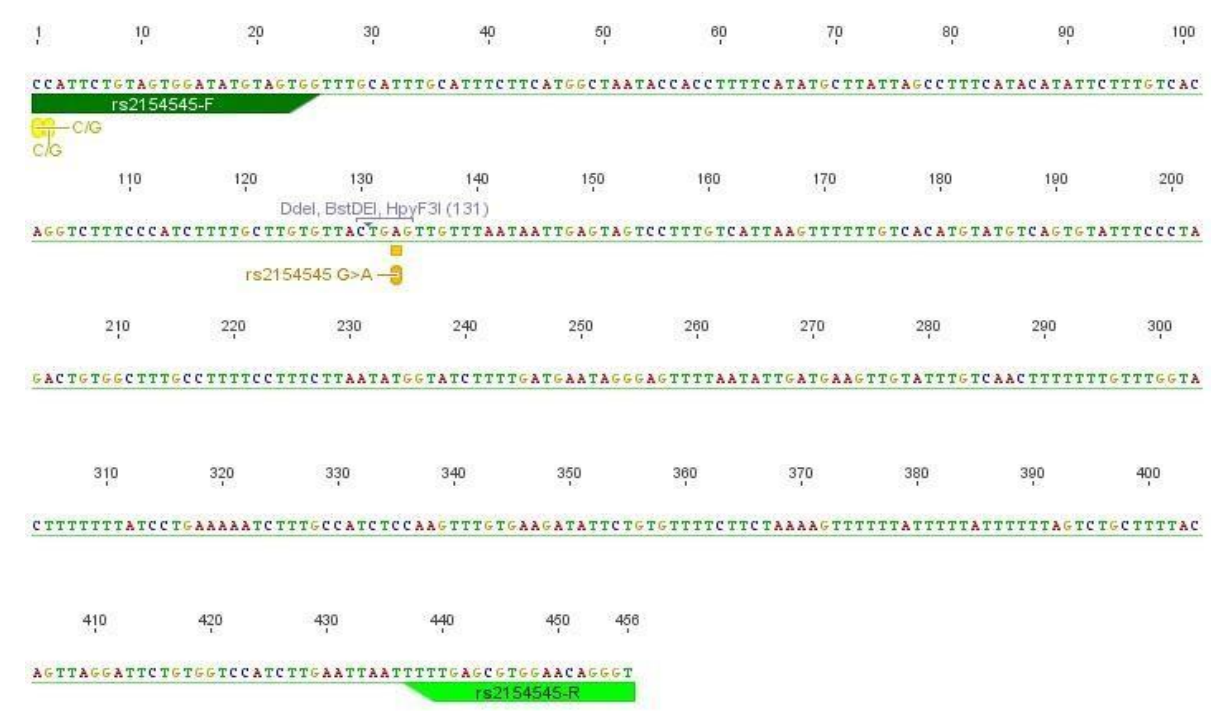

Fig. 1. RFLP-PCR map of DYRKIA (NCBI accession number: NG_009366.1) for rs2154545 (G>A) mutant allele with DdeI restriction site.

PCR was performed under the following conditions: (1) initial denaturation at $95^{\circ} \mathrm{C}$ for 5 minutes; (2) 35 cycles of denaturation at $94^{\circ} \mathrm{C}$ for 1 minute, annealing at $53^{\circ} \mathrm{C}$ for 30 seconds, and elongation at $72^{\circ} \mathrm{C}$ for 30 seconds; and (3) final elongation at $72^{\circ} \mathrm{C}$ for 10 minutes. The PCR product was examined using $1.5 \%$ agarose gel electrophoresis with DNA GelRed stain in GelDoc. The Rs 2154545 PCR product was 456 bp in length.

The PCR product was cut by $1 \mathrm{U}$ of DdeIrestriction enzyme (restriction site $=\mathrm{C}^{\wedge} \mathrm{TN} \underline{\mathrm{AG}}$ ) at $65^{\circ} \mathrm{C}$ for 2 hours. After that, the restriction result was examined using $1.5 \%$ agarose gel electrophoresis with GelRed DNA stain in GelDoc. Samples from individuals with the wildtype rs2154545 genotype (GG allele) resulted in a DNA band that was $456 \mathrm{bp}$ in length. In contrast, samples from individuals with the mutant heterozygote rs2154545 genotype (GA allele) produced $456 \mathrm{bp}, 326 \mathrm{bp}$, and $130 \mathrm{bp}$ DNA bands, and samples from individuals with the mutant homozygote rs2154545 genotype (AA allele) produced $326 \mathrm{bp}$ and $130 \mathrm{bp}$ DNA bands.

\section{Results}

Every subject in this study had trisomy 21, confirmed by karyotyping analysis. Of the 39 subjects, $64.1 \%(n=25)$ were male, $71.8 \%$ were greater than or equal to 10 years old, $74.4 \%$ were born from a mother who was greater than or equal to 35 years old, and $63.1 \%$ had mildborderline intellectual disabilities (Table 1). 
Table 1. Study Subjects Characteristics.

\begin{tabular}{lcc}
\hline Characteristic & $\mathrm{f}=39$ & $\%$ \\
\hline 1. Gender & & \\
* Male Trisomy & 25 & 64.1 \\
* Female Trisomy & 14 & 35.9 \\
2. Age & 11 & 28.2 \\
$\leq 10$-year-old & 28 & 71.8 \\
> 10-year-old & & \\
3. Mother's age & 10 & 25.6 \\
$\leq 35$-year-old & 29 & 74.4 \\
$>$ 35-year-old & & \\
4. Nutrition status & 13 & 43.6 \\
$*$ Underweight & 18 & 30.8 \\
* Norm weight & 8 & 25.6 \\
* Overweight & &
\end{tabular}

\subsection{Karyotyping Analysis}

Blood samples were collected from all subjects and placed in a heparin-coated vacutainer and cultured during an incubation period of 3 to 4 days. The cells were then harvested and the harvested products were deposited onto to a glass slide and left for 1 to 3 days. After that, the banding procedure was carried out. The banding result was analyzed and six metaphase cells were selected for karyotyping. In total, 47 chromosomes, XY, trisomy 21 (male) and 47 chromosomes, XX, trisomy 21 (female) were acquired (Figure 2).

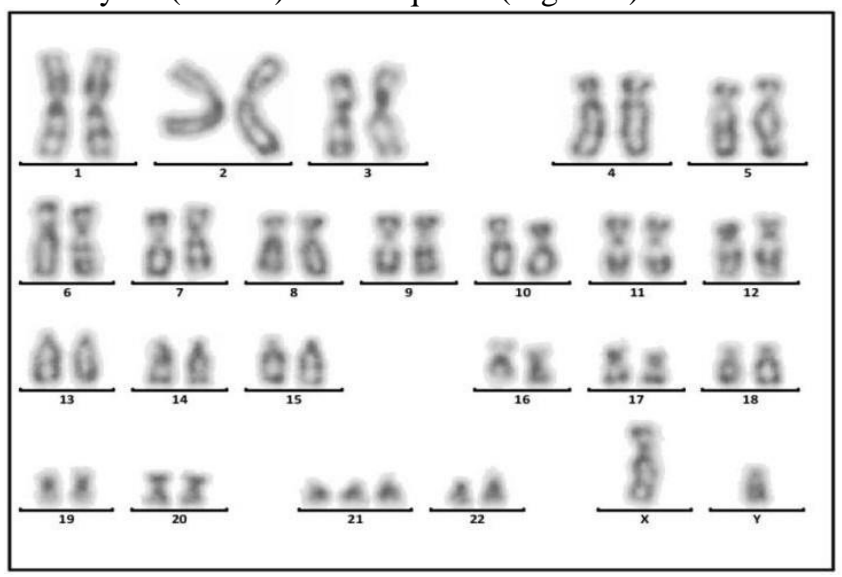

Fig. 2. DS 01 karyotyping result with 3 copies of 21 st chromosome and $X Y$ sex chromosome which implies that DS01 patient is a male.

\subsection{DNA Extraction (Genomic DNA Purification)}

DNA extraction was carried out by following the instruction manual from Genomic DNA Mini Kit (Geneaid), and the sample was examined by gel electrophoresis. Every sample produced a DNA band (Figure 3), which implies that the genomic DNA was successfully isolated. 


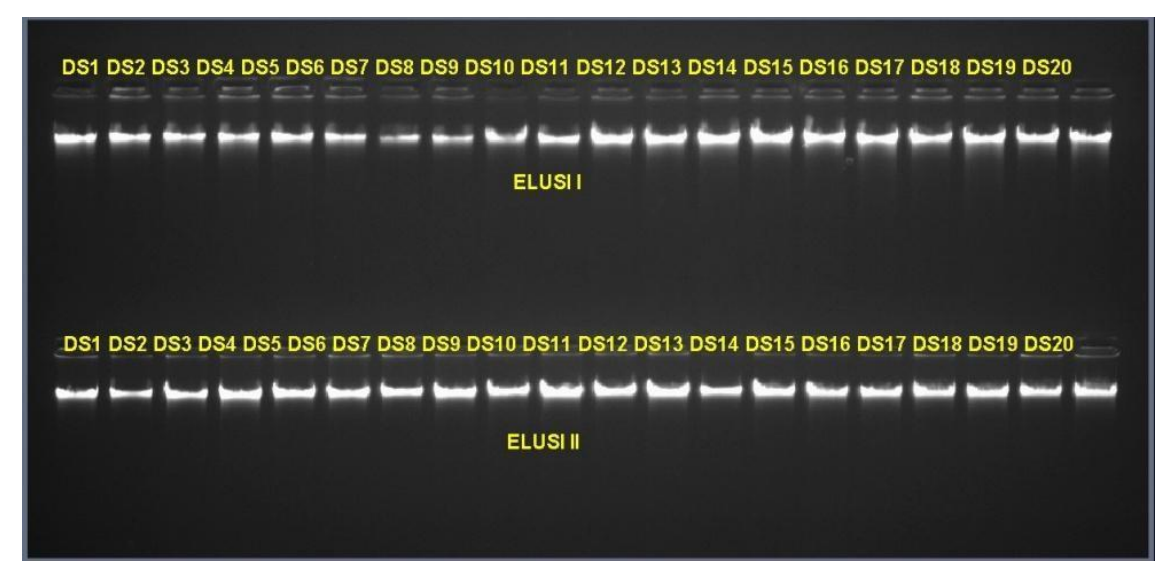

Fig. 3. Electrophoresis result of Isolated DNA from DS1 to DS20 patients. PCR Genotyping rs 2154545 (G>A) Result.

After completion of the PCR and restriction enzyme procedures, DNA samples from four people (DS32, DS15, DS39, and DS31) were separated into three DNA bands - $456 \mathrm{bp}, 326 \mathrm{bp}$, and $130 \mathrm{bp}$ - which implies that the GA allele (mutant heterozygote) was present in those subject. The DNA sample from DS40 DNA was separated into two bands - $326 \mathrm{bp}$ and $130 \mathrm{bp}-$ which implies the AA allele (mutant homozygote). The DNA samples from DS26, DS27, and DS11 only produced one band (456 bp), which implies the GG wild-type allele (Figure 5).

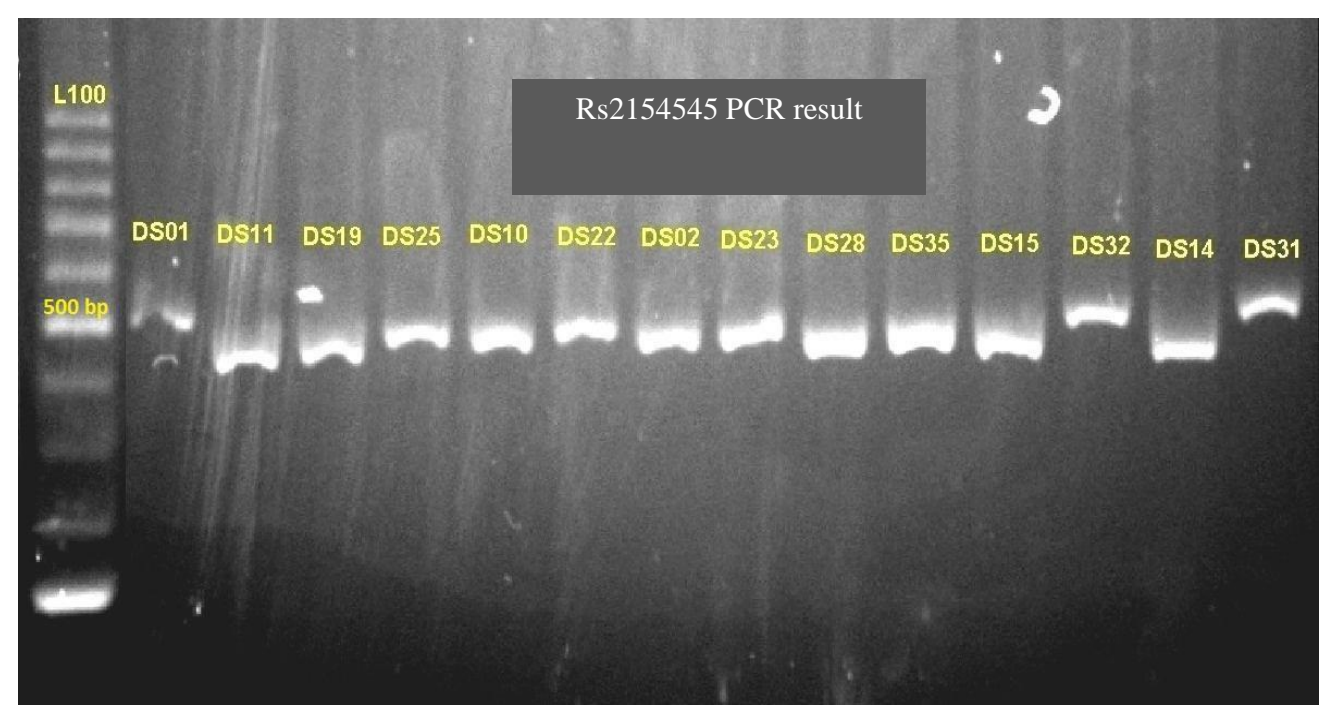

Fig. 4. Electropherogram of DS subject's rs2154545 PCR. Rs2154545 (G>A) PCR product was 456 bp band. 


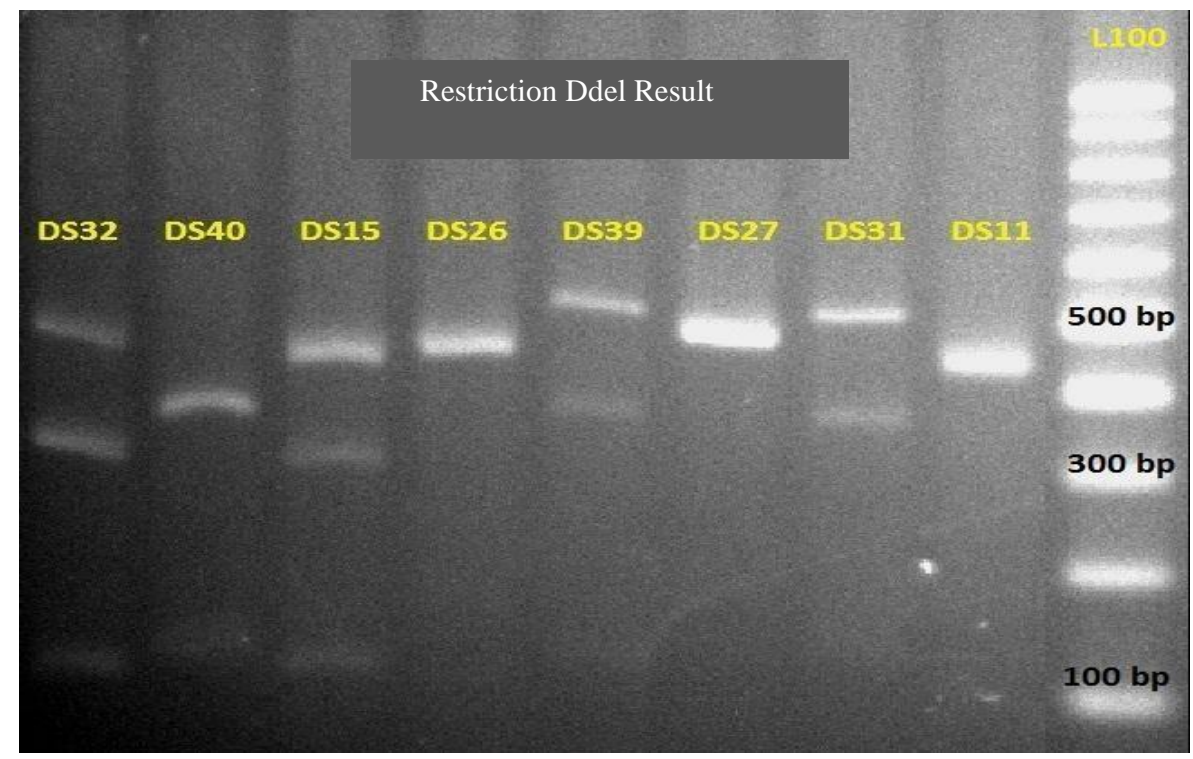

Fig. 5. Electropherogram of Ddel restriction enzyme cut of DS subject's rs2154545. Rs2154545 (G>A) PCR product was 456 bp band.

\subsection{Sequencing}

The PCR product from a few samples was purified and sent to Macrogen, South Korea, for sequencing. Below is the sequencing result of DS22.

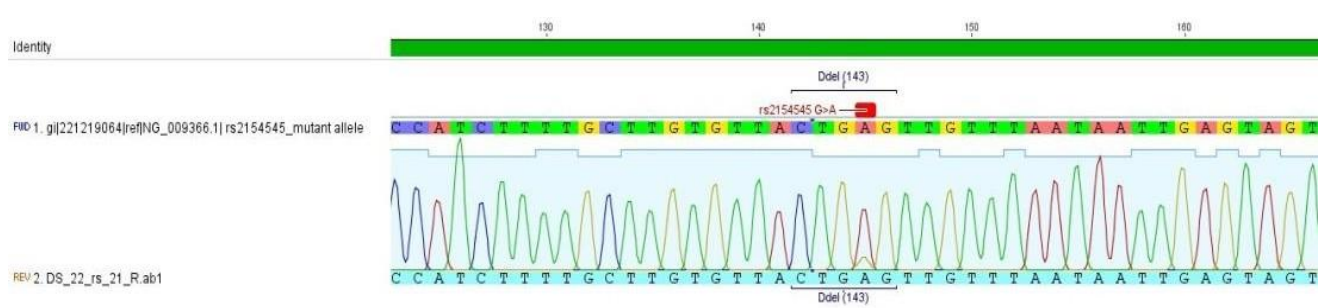

Fig. 6. Contig process of sequencing product of DS 22 DNA sample with forwarding primer for rs 2154545 (G>A) SNP. Sequencing result indicated that DS22 is a mutant heterozygote (GA allele) which in agreement with the RFLP result. 


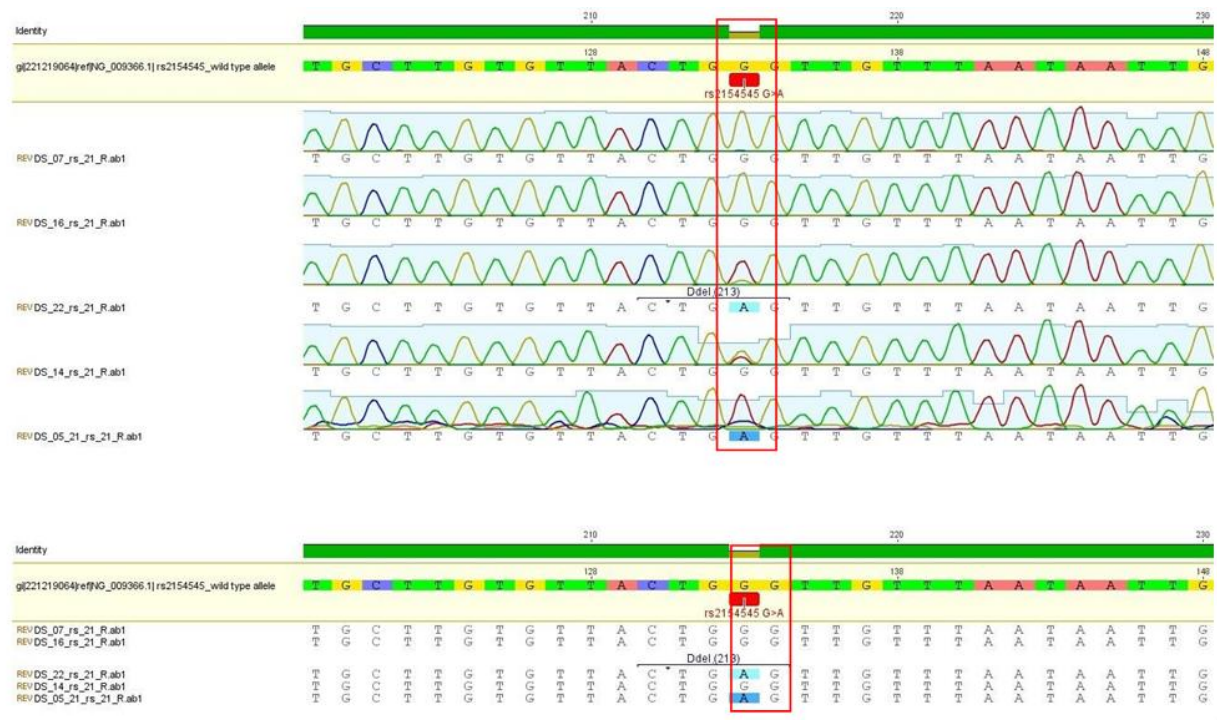

Fig. 7. Multiple alignments from 5 contigs of sequenced samples. Multiple alignments showed that DS 22 and DS 05 have SNP polymorphism rs2154545 in the DyrkIA exon sequence as indicated by nucleotide substitution from G to An in DS 22 and DS 05.

\subsection{Multiple alignment results}

Of all 39 subjects sampled in this study, as many as 17 subjects (43.6\%) had the wild-type allele (GG), two subjects (5.1\%) were mutant homozygotes (AA allele), and 20 subjects $(51.3 \%$ ) were mutant heterozygotes (GA allele) (Table 2). Subjects with the homozygote mutant allele (AA) were equally distributed in the intellectual disability categories of mild-borderline and moderate-severe, but the results were not statistically significant $(p>0.05)$ (Table 3$)$.

Table 2. DyrkIA gene SNP rs2154545 Polymorphism

\begin{tabular}{lll}
\hline SNP rs2154545Polymorphism & F & $\%$ \\
\hline Wild Type (GG allele) & 17 & 43.6 \\
Mutant Homozygote (AA allele) & 2 & 5.1 \\
Mutant Heterozygote (GA allele) & 20 & 51.3 \\
\hline
\end{tabular}

Table 2 has heterozygote mutant $51.3 \%$

Table 3. DyrkIA gene polymorphism rs 2154545 relationship with Mental Retardation level

\begin{tabular}{|c|c|c|c|c|c|c|c|}
\hline \multirow{3}{*}{ SNP rs2154545 } & \multicolumn{4}{|c|}{ Mental retardation } & \multirow{2}{*}{\multicolumn{3}{|c|}{ Total }} \\
\hline & \multicolumn{2}{|c|}{ Mild-Borderline } & \multicolumn{2}{|c|}{ moderate-severe } & & & \\
\hline & $f$ & $\%$ & $\mathrm{~F}$ & $\%$ & $\mathrm{f}$ & $\%$ & $\mathrm{P}$ \\
\hline Wild Type & 9 & 52,9 & 8 & $47.1 \%$ & 17 & 100 & \multirow{3}{*}{0.355} \\
\hline Homozygote & 1 & 50 & 1 & 50 & 2 & 100 & \\
\hline Heterozygot & 15 & 74 & 5 & 25 & 20 & 100 & \\
\hline Total & 25 & 64.1 & 14 & 35.9 & 39 & 100 & \\
\hline
\end{tabular}


Table 3. has heterozygote mutant relationship with the mild borderline of mental retardation

\section{Discussion}

This study included 39 subjects who have been diagnosed with DS (trisomy 21). The syndrome is caused when a gamete cell with an extra copy of chromosome 21 undergoes fertilization with a normal gamete cell, resulting in a zygote with 47 chromosomes $(46 \mathrm{XX}+21$ and $46 \mathrm{XY}+21)$. This abnormality occurred in $90 \%-95 \%$ of all observed cases [8] [9]. In this study, 25 subjects $(65.1 \%)$ were male and 14 subjects were female $(35.9 \%)$. This proportion is in accordance with results reported by Johnson (2006) in which more male babies were born with DS compared to female babies. [10]

The prevalence of intellectual disabilities in people with DS is around 2-3\% of all cases. Most people $(85 \%)$ who have been diagnosed with DS have intellectual disabilities that are considered to be mild, while $0.3 \%-0.5 \%$ have intellectual disabilities that are considered to be severe [11]. Mild intellectual disabilities are likely to occur 7-10 times more frequently compared to moderate and severe intellectual disabilities. Although the physical appearance of DS is easily identified, intellectual disability is the most consistent identifier in DS, and the average IQ of many people with this diagnosis equal to 50 [10]. This study observed $64.1 \%$ people with mild-borderline intellectual disability and $35.9 \%$ with moderate-severe intellectual disability.

In the present study, we observed16 males diagnosed with DS who had mild-borderline intellectual disabilities and 9 males with moderate-severe intellectual disabilities. In the females with DS, 10were considered to have mild-borderline intellectual disabilities and 4 males with moderate-severe intellectual disabilities. Statistical analysis showed that there were no significant relationships between the severity of intellectual disability and gender $(p>0.05)$.

As much as 6 under 10-year-old subjects suffered mild-borderline intellectual disability and 5 subjects in the same age group had a moderate-severe intellectual disability. 20 subjects, older than 10-year- old, had a mild-borderline intellectual disability, while 8 subjects in the same age group had a moderate-severe intellectual disability. Statistical analysis didn't reveal any significant relationship between age and intellectual disability level ( $p>0.05)$.

Based on a study conducted in 2000, people with intellectual disabilities were identified in 12 of every 1,000 school-aged children, or one in every 83 children [11]. Intellectual disability mainly occurs in older people. These previously reported statistics are consistent with the findings of our, in which 11 subjects $(28.2 \%)$ who were less than 10years old had an intellectual disability compared to. 28 subjects $(71.8 \%)$ who were greater than or equal to10years old. As previously stated, our study identified 29 people $(74.4 \%)$ who had mothers who were 35 years or older when giving birth to their child with DS. According to other authors, DS cases occur in one of every 700 births from mothers who are 35years old or older, and the prevalence of DS births increases as the mother's age increases [12][9]. A study in South Africa also found a similar result.

DS can also occur as a result of genetic mutation. Some twohypotheses explain the etiology of DS as the result of mutation: (1) the instability hypothesis that describes the loss of chromosome stability; and (2) the gene dose hypothesis that describes the overexpression of a particular gene. Our study identified the SNP rs2154545 polymorphism on the DyrkIA gene. The wild-type form (GG allele) was observed in 17 samples (43.6\%), the mutant homozygote form (AA allele) in 2 samples (5.1\%), and theheterozygote mutant form in 20 samples $(51.3 \%)$. 
In general, there were 22 rs2154545 SNP mutants (56.4\%) of theDyrkIA gene. This result implies that DyrkIA polymorphisms have a role in the pathogenesis of DS. DyrkIA overexpression has the potency to damage the brain and cause intellectual disability.

In humans, the DyrkIA gene is located on the HSA 21 loci, the long arm of the 21q22.3 chromosome; this region is the critical site of DS and is usually called DScritical region (DSCR). DSCR, which is comprised of $5.4 \mathrm{Mb}$ and 30 genes, has been characterized by phenotypegenotype correlation studies using samples from people diagnosed with partial trisomy 21 [14][5]. Some studies have shown that DyrkIAgene has an influence on the development of the central nervous system, such as neuron proliferation, neurogenesis, differentiation, cell death, and synapse plasticity. The overexpression of this gene in the brain of a fetus with DS supports the hypothesis that DyrkIAplays a role in e neurodevelopment and the cognitive deficits of people with DS [4]. In our study sample, people with DS who were heterozygous for the SNP rs2154545 polymorphism were mainly found to have mild-borderline intellectual disabilities $(74.0 \%)$, while half of the sample who were homozygous for the polymorphism had mildborderline intellectual disabilities and half had moderate-severe intellectual disabilities. However, no findings reached statistical significance.

A study of seniors with Alzheimer's disease in Madrid, Spain, found variations of the DyrkIA gene (SNP polymorphisms rs117001483, rs2835740, rs1137600, rs2154545, rs8132976, and rs2835762) that did not reach statistical significance [15]. A study in a Chinese population found a relationship between mild cognitive amnestic disturbance and SNP polymorphisms in three systems that relate to Alzheimer's pathogenesis. Those three systems were the amyloid cascade pathway, Tau metabolism, and cholesterol [16]

In conclusion, the results of our study show that there are an observable SNP rs 2154545 polymorphism on the DrykIA gene has heterozygote mutant (Allele GA) that causes the most common clinical manifestation of this overexpression is a mild-borderline intellectual disability in people with DS.

\section{References}

[1] Chen H (1994)Down syndrome. Growth42,157-62

[2] Capone GTW(2001) Down syndrome: advances in molecular biology and the neurosciences. Dev behavi pediatr, 22.

[3] Dutta S, Gangopadhyay PK, Mukhopadhyay K (2005) Molecular aspects of down syndrome. Indian pediatr $42,339-44$.

[4] Tejedor FJ (2011) MNBDYRK1A as a multiple regulator of neuronal development. FEBS Journal 278, 223-235.

[5] Kida E, Jarzabek K, Palminiello S, Albertini G, Rabe A, Hwang YW, and Golabek A (2011) Form of dual- specificity tyrosine-(Y)- phosphorylation regulated kinase $1 \mathrm{~A}$ nonphosphorylated at tyrosine 145 and 147 is enriched in the nuclei of astroglial cells, adult ippocampal progenitors, and some cholinergic axon terminals. Neuroscience 195, 112-127

[6] Vasconcelos MM (2004) Mental retardation. J pediatr 80, s71-82.

[7] Barkoukis A, and Reiss NS (2008) Dombeck M. Childhood disorder: mental retardation. Http://www.mentalhelp.net

[8] Khalil A (2006) Screening for down syndrome. J obstet gynecol india 56, 205-11.

[9] Descartes MCA (2007) Cytogenetics. In: Kliegman RM, Jenson HB, Stanton BF (ed.) Nelson textbook of pediatrics. 18 ed. Philadelphia: Saunders Elsevier.

[10] Johnson CP(2006) Mental retardation: management and prognosis. Peds in review 27, 249-56.

[11] CDC (2010) Intellectual disabilities among children. Http://www.cdc.gov/intellectualdisabilities.

[12] Morris JK, and Alberman E (2002) Revised estimates of the maternal age specific live birth prevalence of down's syndrome. J med screen 9, 2-6. 
[13] Park P, Chung KC(2009) Two key genes closely implicated with the neuropathological characteristics inDown syndrome: DYRK1A and RCAN1.BMB Rep.42(1), 6-15.

[14] Park P, Chung KC(2009) Two key genes closely implicated with the neuropathological characteristics inDown syndrome: DYRK1A and RCAN1.BMB Rep.42(1), 6-15.

[15] Higuera JLV,Juan PS,Rodriguez ER, Ignacio M, Ana P, Ana F, Isabel S, Fernando V, José B, María J B, and Onofre C (2009) DyrkIA genetic variants are not linked to Alzheimer's disease in a Spanish case-control cohort.BMC Med Genet 2009,10-129

[16] Liu X,Yue Ch,Xu Z,Pu M,Yu H,Yongmei S, Liying Z, Xiaohui X, and Zhijun Z (2012) Association study of candidate gene polymorphisme with amnestic mild cognitive impairment in a Chinese population.PloS One 7(7), e41198 